\title{
Diagnostic Yield Variation with Colonoscopy among Pediatric Endoscopists
}

\author{
Jay A. Hochman ${ }^{a}$ Janet Figueroa ${ }^{b}$ Emily Duner ${ }^{c}$ Jeffery D. Lewis ${ }^{a}$

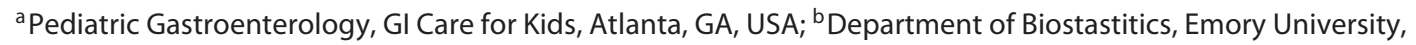 \\ Atlanta, GA, USA; ${ }^{C}$ College of Arts and Sciences, Northwestern University, Chicago, IL, USA
}

\begin{abstract}
Keywords
Ileal intubation · Colonoscopy · Provider variation .

Diagnostic yield
\end{abstract}

\begin{abstract}
Background: The primary aim of our study was to determine provider variation in diagnostic yield in a pediatric endoscopy center. Secondary aims were to examine ileal intubation rates as well as procedural complications at the provider level. Methods: A retrospective review of sequential pediatric patients who underwent a colonoscopy, completed by June 2018, determined the rates of endoscopically abnormal (EA) and isolated histologically abnormal (IHA) colonoscopies; the overall diagnostic yield was the combination of EA and IHA. Results: In total, 374 charts were reviewed. This study found high variability in diagnostic yield among the 16 clinicians ranging from as low as $22 \%$ to as high as $86 \%$ ( $p=$ 0.11 ) with an overall diagnostic yield of $48 \%$ for colonoscopy; excluding follow-up colonoscopies, the diagnostic yield was $42 \%$. Abnormal calprotectin and abnormal blood tests were associated with higher diagnostic yields of 83 and $65 \%$, respectively, compared with symptoms such as abdominal pain, diarrhea, and rectal bleeding which had yields of 39,43 , and $61 \%$. lleal intubation rates averaged $90 \%$ (range $63-100 \%, p=0.06)$. In patients with a normal colon, there were $21(6 \%)$ patients with an EA ileum and an additional 16 (4\%) with an IHA ileum. Prep quality was rated excellent, good, or average in $97 \%$. In addition, there was a low rate of
\end{abstract}

serious complications ( 1 of 374 ). Conclusions: This study highlights the individual variability in diagnostic yield and ileal intubation rates in a pediatric gastroenterology practice. Goals for pediatric endoscopy could include ileal intubation rates of $>90 \%$ and provider diagnostic yields of $>40 \%$.

(c) 2019 S. Karger AG, Basel

\section{Introduction}

With pediatric gastroenterology endoscopic procedures, there are little data regarding variability in provider performance with regard to diagnostic yield [1-4]. A recent NASPGHAN clinical report [3] sought to provide parameters for quality improvement in pediatric endoscopy. "A high-quality endoscopy has been defined as an examination in which patients receive an indicated procedure, correct and relevant diagnoses are recognized or excluded, any therapy provided is appropriate, and all steps that minimize risk have been taken." Specific measures include patient education, timeliness, bowel preparation, ileal intubation, adverse events, and patient satisfaction.

In clinical practice, there is wide variation in indications for colonoscopy and the frequency of colonoscopy per provider. Careful selection of patients can help improve diagnostic yield and minimize the risk of low value procedures. A normal colonoscopy has not been shown to improve outcomes in children with functional gastrointestinal diseases [5].

\section{KARGER}

(c) 2019 S. Karger AG, Basel

karger@karger.com

www.karger.com/ddi
Jay A. Hochman, MD

Pediatric Gastroenterology

GI Care for Kids, 993-D Johnson Ferry Road, 440

Atlanta, GA 30342 (USA)

E-Mail jjhochman@ gmail.com 
As part of our efforts in establishing quality metrics in our outpatient endoscopy center, we aimed to identify the variation in diagnostic yield among the 16 clinicians in our pediatric private practice group. Secondary aims were to examine ileal intubation rates as well as procedural complications at the provider level.

Our goal is for every pediatric patient to have a highquality endoscopy designated by a good indication for the procedure, good technique, and appropriate follow-up. Even though diagnostic yield is not used as a quality metric [1-3], it is likely important as a measure of high value care. This single-center retrospective chart review of an ambulatory pediatric endoscopy center cohort sought to determine the provider variation in diagnostic yield.

\section{Methods}

After obtaining approval from our Institutional Review Board, we performed a retrospective chart review of sequential pediatric patients who underwent a colonoscopy at our outpatient pediatric endoscopy center. Specifically, for each of 16 clinicians, we attempted to identify the most recent 25 charts of patients who had a colonoscopy. Charts were selected by review of the ambulatory center's schedule. All procedures were completed prior to June 2018.

In total, 374 charts were reviewed instead of 400 . This was due to several factors. The decision to identify 25 charts was based on the goal of identifying a similar number of patients in each of the 16 providers at the ambulatory center. Two physicians had completed only 22 colonoscopies at the time of this review; one because of joining the group in the preceding year and one due to less frequent use of colonoscopy over approximately 3 years. After review, there were several charts pulled in which patients did not have a colonoscopy; these were misclassified in the scheduling system.

Prep quality in this study was a subjective assessment by the endoscopist as determined by the operative report. All patients received the same prep instructions which included polyethylene glycol followed by a sodium phosphate enema; the latter could be skipped if stools were clear.

After obtaining demographic information, each chart was examined for indications, number of office visits before colonoscopy, testing prior to procedure, and treatments prior to colonoscopy. Blood tests and stool calprotectin were considered abnormal based on the performing commercial laboratory. Other variables that were reviewed included whether this was an isolated colonoscopy or a colonoscopy-esophagogastroduodenoscopy (EGD; panendoscopy), whether the procedure was a first procedure or a followup, pathology results, and complications following procedure. With regard to complications, each chart was reviewed to determine if there were any emergency room visits, hospitalizations, or office visits within a week following the procedure.

With regard to indications, the term "isolated abdominal pain" was used when no other major indications, such as weight loss, diarrhea, or rectal bleeding, were present. Some patients with isolated abdominal pain had a family history of inflammatory bowel disease (IBD). Due to the variability of documentation of family history, this factor was not specifically identified.
Abnormal histology was determined by review of the pathology report. Each pathology report was generated from one of 2 pathologists.

When reviewing charts for diagnostic yield, we considered the finding of gross abnormalities in the appearance of the mucosa as endoscopically abnormal (EA) and in those with normal mucosa but abnormal pathology as isolated histologically abnormal (IHA). The focus on EA was based on several factors. For IBD, the Paris classification relies on gross findings to establish extent of disease [6]. In addition, vague nonspecific pathologic findings are frequently evident and often irrelevant, particularly with EGD. Several trivial findings including lymphoid hyperplasia, internal hemorrhoids, and pinworms were not considered EA.

\section{Statistics Information}

All statistical analyses were conducted using SAS 9.4 (Cary, NC, USA). Descriptive statistics using medians and interquartile ranges for continuous variables or counts and frequencies for categorical variables were analyzed. Continuous data were compared using Wilcoxon rank-sum tests and categorical variables were compared using chi-square or Fisher's exact tests (for cell counts $<5)$. Variability between all 16 providers in normal colonoscopy yield, whether the ileum was reached, and type of procedure used (colonoscopy vs. combined with EGD) was assessed using generalized linear mixed models with a subject-specific random intercept for providers. Z-tests from the modeled random intercepts were used to determine the presence of significant variability between providers. Statistical significance for all hypothesis testing was determined at the 0.05 level using two-tailed $p$ values.

\section{Results}

\section{Patient Characteristics}

In total, 374 colonoscopies completed by 16 pediatric gastroenterologists were reviewed. The demographics and characteristics are detailed in Table 1 . Most patients $(n=$ $318,85 \%$ ) who underwent a colonoscopy underwent a combination procedure with EGD; 56 (15\%) patients underwent an isolated colonoscopy. All patients received general anesthesia with propofol administered by an anesthesiologist.

\section{Workup}

The majority of patients underwent testing prior to endoscopy. Three hundred thirteen (84\%) patients had blood tests obtained, and 159 (43\%) patients had a stool calprotectin. Among those with testing, 86 (28\%) had an abnormality in blood, and $73(46 \%)$ had an abnormal stool calprotectin. There were 45 patients with an abnormal calprotectin and normal blood tests. In addition, 145 (39\%) had stool tests done to evaluate for stool pathogens; the frequency of stool pathogen testing is identical to the frequency of patients with diarrhea as an indication for colonoscopy (Table 2). 
Table 1. Overall summary characteristics, $n=374$ patients

\begin{tabular}{|c|c|}
\hline Patient demographics & All, $n(\%)$ \\
\hline \multicolumn{2}{|l|}{ Gender } \\
\hline Male & $171(45.7)$ \\
\hline Female & $203(54.3)$ \\
\hline Patient age, years, median (25th-75th) & $14(11-17)$ \\
\hline \multicolumn{2}{|c|}{ Time from 1st office visit until procedure date, weeks } \\
\hline$<2$ & $58(15.5)$ \\
\hline $2-4$ & $74(19.8)$ \\
\hline $4-8$ & $71(19.0)$ \\
\hline $8-16$ & $38(10.2)$ \\
\hline $16-52$ & $36(9.6)$ \\
\hline$>52$ & $97(25.9)$ \\
\hline \multicolumn{2}{|l|}{ Procedure } \\
\hline Colonoscopy only & $56(15.0)$ \\
\hline EGD - colonoscopy & $318(85.0)$ \\
\hline \multicolumn{2}{|l|}{ 1st or follow-up colonoscopy } \\
\hline 1 st & $302(80.8)$ \\
\hline Follow-up & $72(19.2)$ \\
\hline \multicolumn{2}{|l|}{ Workup prior to endoscopy } \\
\hline \multicolumn{2}{|l|}{ Hemoglobin } \\
\hline Low & $53(14)$ \\
\hline Normal & $258(69)$ \\
\hline Not ordered & $63(17)$ \\
\hline \multicolumn{2}{|l|}{ Sedimentation rate } \\
\hline Elevated & $38(10)$ \\
\hline Normal & $214(57)$ \\
\hline Not ordered & $122(33)$ \\
\hline \multicolumn{2}{|l|}{ Albumin } \\
\hline Low & $18(5)$ \\
\hline Normal & $270(72)$ \\
\hline Not ordered & $86(23)$ \\
\hline \multicolumn{2}{|l|}{ C-reactive protein } \\
\hline High & $36(10)$ \\
\hline Normal & $223(60)$ \\
\hline Not ordered & $115(30)$ \\
\hline \multicolumn{2}{|l|}{ Any abnormal blood test } \\
\hline Abnormal & $86(23)$ \\
\hline All normal & $227(61)$ \\
\hline Not ordered & $61(16)$ \\
\hline \multicolumn{2}{|l|}{ Stool calprotectin } \\
\hline Elevated & $73(20)$ \\
\hline Normal & $86(23)$ \\
\hline Not ordered & $215(57)$ \\
\hline \multicolumn{2}{|l|}{ Prep quality } \\
\hline Excellent & $83(22.3)$ \\
\hline Good & $230(61.8)$ \\
\hline Adequate & $47(12.6)$ \\
\hline Poor & $12(3.2)$ \\
\hline
\end{tabular}

EGD, esophagogastroduodenoscopy.

\section{Prep Quality}

Overall, clinicians encountered a poor prep in only 12 (3\%) colonoscopies; the prep was considered excellent in $83(22 \%)$, good in $230(62 \%)$, and adequate in 47 (13\%; Table 1).

Colonoscopy Yield among Pediatric Endoscopists
Table 2. Indications for procedure, $n=374$ patients

\begin{tabular}{|c|c|}
\hline Indications (not mutually exclusive) & Number (\%) \\
\hline Rectal bleeding & $95(25)$ \\
\hline Abdominal pain (including isolated abdominal pain) ${ }^{1}$ & $258(69)$ \\
\hline Isolated abdominal pain only ${ }^{1}$ & $46(12)$ \\
\hline Weight loss/poor growth & $75(20)$ \\
\hline Diarrhea & $145(39)$ \\
\hline Abnormal blood test (ESR, CRP, albumin, hemoglobin) ${ }^{2}$ & $86(23)$ \\
\hline Abnormal calprotectin ${ }^{3}$ & $73(20)$ \\
\hline Abnormal calprotectin and normal blood labs ${ }^{4}$ & $38(10)$ \\
\hline Follow-up IBD & $33(9)$ \\
\hline Other ${ }^{5}$ & $18(5)$ \\
\hline
\end{tabular}

\footnotetext{
${ }^{1}$ Isolated abdominal pain: abdominal pain without presence of abnormal blood lab, rectal bleeding, diarrhea, or weight loss/poor growth.

${ }^{2} n=61$ not tested for any blood test: ESR, CRP, albumin, or hemoglo-

${ }^{3} n=215$ not tested for calprotectin.

${ }^{4} n=226$ did not have tests for calprotectin and blood test.

${ }^{5}$ Other includes: congenital hypertrophy of retinal pigment epithelium (CHRPE), and abnormal imaging.

IBD, inflammatory bowel disease; ESR, erythrocyte sedimentation rate; $\mathrm{CRP}, \mathrm{C}$-reactive protein.
} bin.

\section{Indications}

In the majority of patients, there were a combination of indications for the colonoscopy (Table 2). While abdominal pain was present in 258 (69\%) of patients who underwent colonoscopy, only $12 \%$ had isolated abdominal pain without another major indication.

\section{Diagnostic Yield}

There was considerable variability of diagnostic yield among clinicians, though it did not reach statistical significance $(p=0.11)$. As evident in Figure 1, diagnostic yield (combined EA and IHA) was as low as $22 \%$ to as high as $86 \%$ with an overall diagnostic yield of $48 \%$ for colonoscopy; the median value for diagnostic yield was also $48 \%$. When excluding the follow-up cases, the average diagnostic yield was $42 \%$.

Among patients with abnormal laboratory values, the diagnostic yield was higher than for those with other indications based on symptoms (Fig. 2). In those with abnormal blood tests, the colonoscopy results were EA in 56 of $93(60 \%)$ and IHA in an additional $5(5 \%)$. In those with an abnormal stool calprotectin, 53 (73\%) had an EA colonoscopy and an additional 7 (10\%) had an IHA colonoscopy.

The symptom with the highest diagnostic yield, when combining both EA and IHA, was rectal bleeding at $61 \%$ (Fig. 2). In comparison, diagnostic yield for abdominal pain was $39 \%$, weight loss was $45 \%$, and diarrhea was $43 \%$. Isolated abdominal pain, which indicated that none 


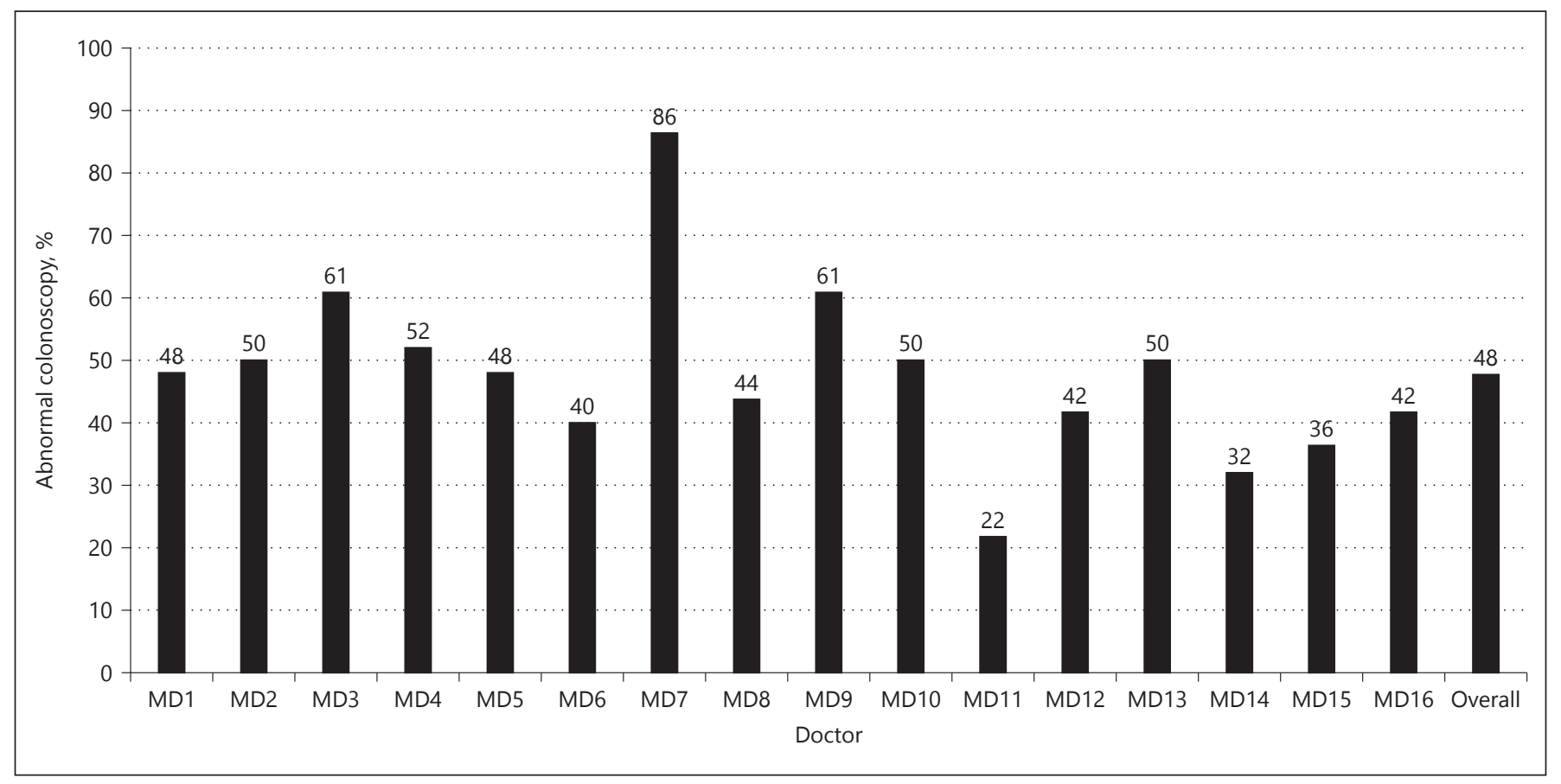

Fig. 1. Colonoscopy diagnostic yield by doctor. The diagnostic yield for colonoscopy varied considerably among the 16 individual physicians. The diagnostic yield was a summary of both EA and IHA. EA, endoscopically abnormal; IHA, isolated histologically abnormal.

of the other major indicators were present, yielded a diagnostic yield of $24 \%$.

\section{Isolated Colonoscopy Compared to Panendoscopy}

As noted in Figure 3, there was variability among clinicians in the frequency of isolated colonoscopy procedure usage, from 0 to $42 \%$. The frequency of having a normal procedure was significantly more common among patients who underwent a panendoscopy procedure compared to patients who underwent an isolated colonoscopy (Table 3). Normal endoscopic appearance of colon and ileum was approximately twice as common in patients undergoing a panendoscopy $(60 \%)$ compared with isolated colonoscopy (27\%). Similarly, when combining gross appearance and histology among patients with panendoscopy, $53 \%$ were normal compared with $26 \%$ of isolated colonoscopy patients.

\section{Ileal Intubation}

The success rate of ileal intubation ranged from 63 to $100 \%$ with a group average of $90 \%$ (Fig. 4); the differences in the rate of ileal intubation did not reach statistical significance $(p=0.06)$. Furthermore, there was not a correlation between ileal intubation rate and clinician expe- rience; however, all physicians in the study had a minimum of 5 years of clinical experience after fellowship.

It is worth noting that some physicians may elect to forego ileal intubation in patients with colonic polyps; though, this effect on ileal intubation is unclear. In our cohort, polyps were present in 27 patients (7\%). Two of the clinicians who achieved a $100 \%$ intubation rate both had 2 patients with polyps.

One reason why ileal intubation is important is related to the diagnostic yields. Among patients with a normal colon, there were 21 (6.4\%) patients with an EA ileum in this cohort and an additional $16(4.3 \%)$ with an IHA ileum. Thus, in total, $10.7 \%$ of the diagnostic yield of the colonoscopy was exclusively from the ileum. Also, we examined the charts of the 16 patients with IHA of the ileum; 6 of these patients had a prior diagnosis of IBD. After at least 1 year of follow-up in all cases, only 1 patient of the remaining 10 patients was determined to have a new diagnosis of IBD; the others were thought to have functional bowel disease.

\section{EGD Findings}

In patients who had panendoscopy, the findings from the EGD were reviewed and are summarized in Table 3. While a grossly normal EGD was identified in $59 \%$, only 


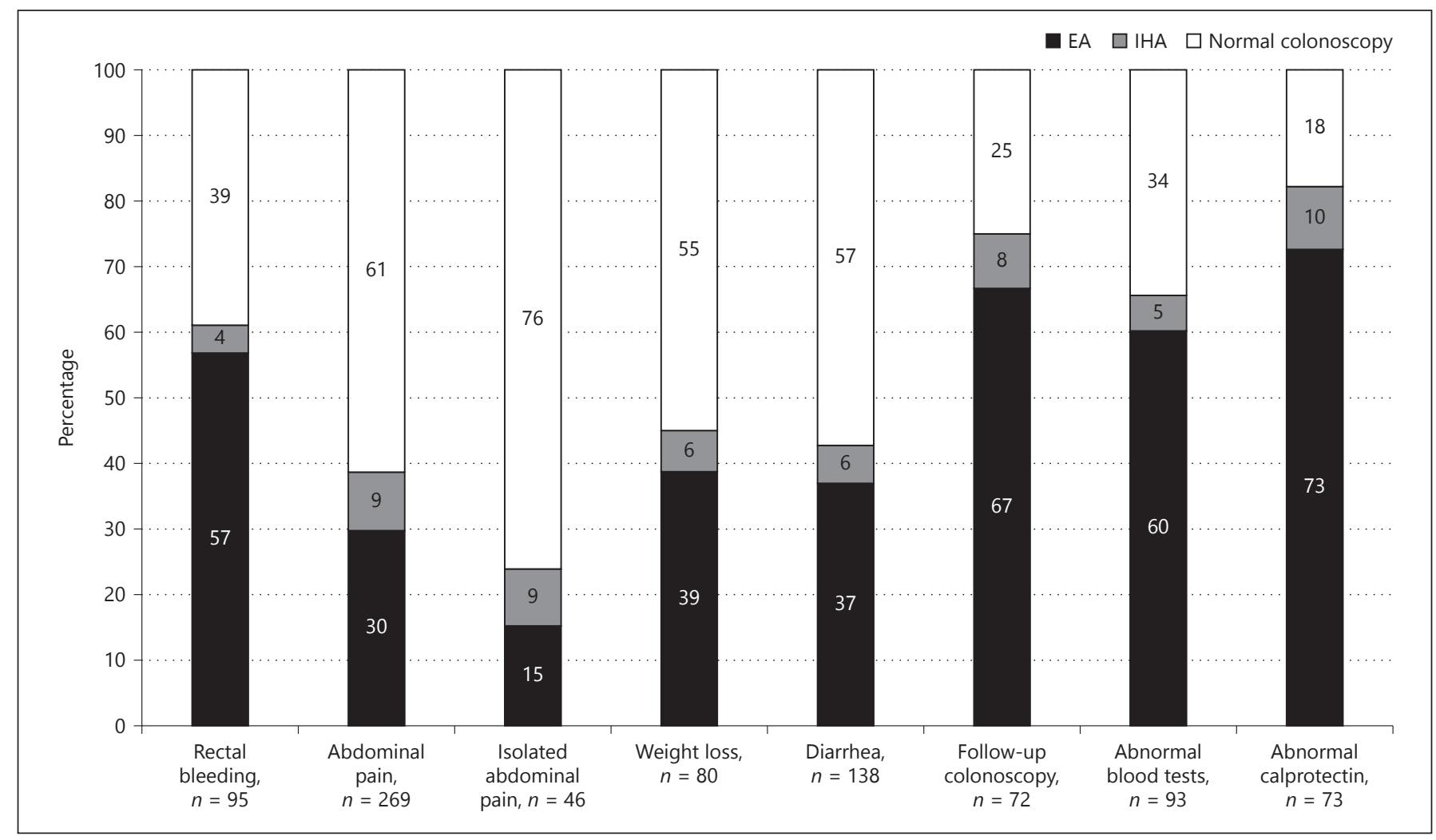

Fig. 2. Colonoscopy yield by indication. The diagnostic yield for colonoscopy varied considerably based on the indication. Findings were classified as either EA based on gross abnormalities in the appearance of the mucosa or as IHA. EA, endoscopically abnormal; IHA, isolated histologically abnormal.

$29 \%$ were considered to have normal histology and normal appearance. Overall, it is noted that EGDs are performed much more frequently than colonoscopies in our patient population. From January 1, 2018, through December 31, 2018, our outpatient center had 1968 EGDs and 780 colonoscopies.

\section{Safety}

The safety of isolated colonoscopy and panendoscopy was excellent in this cohort. Only 1 patient had a significant complication. This patient had bleeding that began a few hours after a polypectomy; this resulted in a hospital admission, blood transfusion, and repeat colonoscopy for control of bleeding.

\section{Discussion}

This retrospective review of an ambulatory pediatric gastroenterology private practice identified high variability in diagnostic yield among the 16 clinicians ranging from as low as $22 \%$ to as high as $86 \%$ with an overall diagnostic yield of $48 \%$ for colonoscopy; the diagnostic yield was $42 \%$ when excluding follow-up cases. In addition, there was high variability in ileal intubation rates with an average of $90 \%$ (range $63-100 \%$ ) and a low rate of serious complications (1 of 374).

To our knowledge, variability by individual physicians in diagnostic yield and ileal intubation rate has not been reported in a large pediatric population previously. It is notable that our overall diagnostic yield of $48 \%$ is similar with other studies [7-13]. Manfredi et al. [8] examined 390 colonoscopies performed by 26 different clinicians. Abnormal gross findings were present in 218 (56\%), whereas $50 \%$ had abnormal pathology. In other pediatric studies, the diagnostic yields for colonoscopy ranged from 33 to $64 \%[9,10,13-15]$.

It is worthwhile to speculate about why there is so much variation in diagnostic yield among clinicians in our practice. Certainly, some variation could be accounted for by differences in patient encounters and some variation could be due to chance related to sample size. 


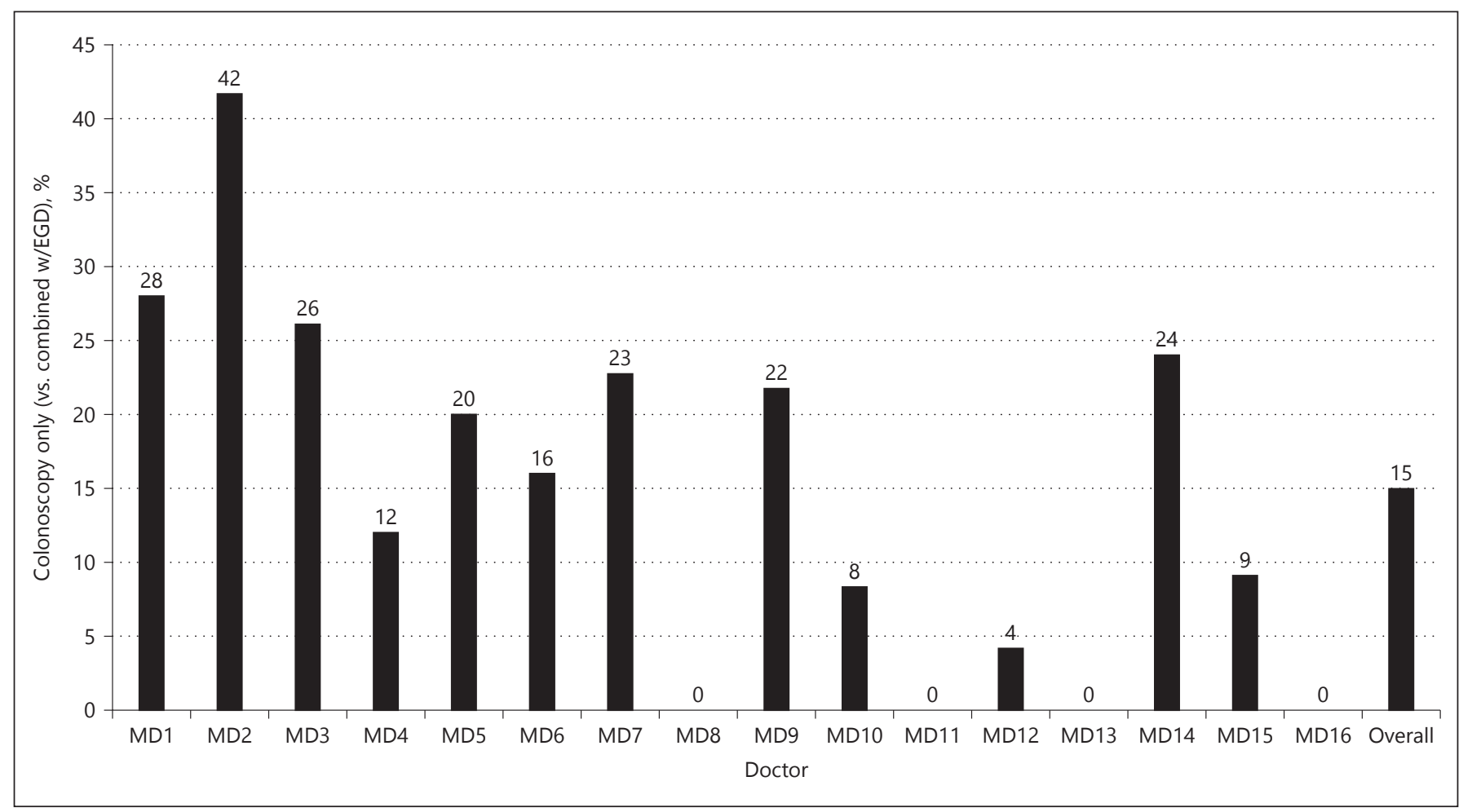

Fig. 3. Variation among physicians in using isolated colonoscopy compared with panendoscopy. This figure shows the frequent variation among 16 individual physicians in the use of isolated colonoscopy or panendoscopy. Overall, only $15 \%$ of patients undergoing a colonoscopy had an isolated colonoscopy and $85 \%$ had a panendoscopy.

Some physicians, based on their interests and reputations, may have subtle differences in the likelihood of organic disease in their individual patient populations. When examining the individual physicians in our study with the highest and lowest yield, there was not a unique patient population; that is, neither physician had a specialized focus in IBD nor functional bowel disease. Thus, it is more likely that the driving factor for the variation in diagnostic yield is related to the threshold for performing an endoscopy in patients with probable functional disorders. In addition, some physicians may be more likely to recommend a colonoscopy for isolated rectal bleeding and some may be more likely to start with laxative therapy. For all of these decisions, the role of parental pressures or physician attitudes cannot be discerned in a retrospective study.

While a negative endoscopic study has not been shown to improve outcomes in patients with functional abdominal pain [5], it is likely that a normal colonoscopy would provide significant reassurance in some specific circumstances including in familial polyposis and in patients at high risk for IBD.
Since the diagnostic yield in our cohort was lower for panendoscopy compared with for isolated colonoscopy, this is likely related to increased selection of panendoscopy in children with functional disorders. In addition, the indications of rectal bleeding and follow-up IBD which had higher diagnostic yield rates than other indications were more likely to result in an isolated colonoscopy.

Our group's average ileal intubation rate of $90 \%$ compares favorably with other studies which have ranged from 52 to $100 \%[7,9,10,13,16-18]$. The pediatric study reporting a $100 \%$ ileal intubation rate involved 6 endoscopists who had $>10$ years of experience (mean 19 years) and more than a thousand prior colonoscopies each [13]. The NASGPHAN clinical report on quality improvement in pediatric endoscopy suggests an ileal intubation goal of $90 \%$ as a general quality metric [3], though noting that this is often not achieved. Our study shows why ileal intubation is important. In our cohort, among patients with a normal colon, there were $21(6 \%)$ with a grossly abnormal ileum and an additional 16 (4\%) with abnormal histology in the ileum. Thus, $>10 \%$ of patients with a normal 
Table 3. Comparisons of colonoscopy yield by isolated colonoscopy compared with panendoscopy

\begin{tabular}{|c|c|c|c|c|}
\hline Normal visual appearance of colon, $n(\%)$ & & & & $<0.001$ \\
\hline Yes & $249(66.6)$ & $22(39.2)$ & $227(71.4)$ & \\
\hline No & $125(33.4)$ & $34(60.7)$ & $91(28.6)$ & \\
\hline \multicolumn{5}{|l|}{ Normal visual appearance of ileum, $n(\%)$} \\
\hline No & $43(11.5)$ & $5(8.9)$ & $38(11.9)$ & \\
\hline Normal visual appearance of colon and ileum, $n(\%)$ & & & & $<0.001$ \\
\hline Yes & $228(60.9)$ & $18(32.1)$ & $210(66.0)$ & \\
\hline No & $110(29.4)$ & $24(42.9)$ & $86(27.0)$ & \\
\hline Ileum not reached & $36(9.6)$ & $14(25.0)$ & $22(6.9)$ & \\
\hline Normal colon and ileum pathology, $n(\%)$ & & & & 0.001 \\
\hline IHA & $32(8.6)$ & $4(7.1)$ & $28(8.8)$ & \\
\hline EA & $146(39.0)$ & $35(62.5)$ & $111(34.9)$ & \\
\hline Normal visual EGD, $n(\%)$ & & & & - \\
\hline Yes & $186(59)$ & - & $186(59)$ & \\
\hline No & $129(41)$ & - & $129(41)$ & \\
\hline Normal EGD pathology, $n(\%)$ & & & & - \\
\hline Yes & $106(33.5)$ & - & $106(33.5)$ & \\
\hline No & $210(66.5)$ & - & $210(66.5)$ & \\
\hline Diagnostic yield EGD, $n(\%)$ & & & & - \\
\hline Normal & $91(28.9)$ & - & $91(28.9)$ & \\
\hline IHA & $95(30.2)$ & - & $95(30.2)$ & \\
\hline EA & $129(41)$ & - & $129(41)$ & \\
\hline
\end{tabular}

$p$ value: chi-square tests (or Fisher's exact if counts $<5$ ) between $C$ and EGD patient groups.

EA, endoscopically abnormal; IHA, isolated histologically abnormal; EGD, esophagogastroduodenoscopy.

endoscopic and histologic evaluation of the colon had abnormalities in the terminal ileum.

While the discrepancy between the gross appearance and histology is well-recognized with EGD [19-21], most reports from pediatric colonoscopy show good correlation between gross findings and histology [8-10]. In one study of 390 colonoscopies, there were 20 patients with abnormal histology with normal visual findings [8]; 8 of the 20 had a known diagnosis of IBD, 4 had symptoms highly suspicious for IBD, and another 3 patients were immunosuppressed due to either bone marrow transplant or nephrotic syndrome. Thus, only 5 patients had the perplexing problem of isolated histologic abnormalities. In the absence of other gross abnormalities, mild ileitis on biopsy presents a situation which requires close follow-up and possibly further investigation to determine whether this has any clinical significance, particularly in patients without previous underlying disease. In our study, only 1 of 10 patients with IHA of ileum was subsequently determined to have new-onset IBD.

Our study showed that 46 patients (12\%) had isolated abdominal pain as the indication for colonoscopy. The lower diagnostic yield of $24 \%$ (15\% EA, 9\% IHA) in this subgroup is in agreement with prior studies $[7,8,10,11$, 16]. However, an important limitation in this finding is the difficulty in identifying some less frequent coexisting indications in a retrospective review, including first degree relatives with IBD, need for fecal microbiota transplantation, and abnormal studies from outside institutions. A recent large retrospective review with 652 colonoscopies showed a $0 \%$ yield when the indication was isolated abdominal pain [16].

In contrast to isolated abdominal pain, our study shows that an abnormal fecal calprotectin was associat- 


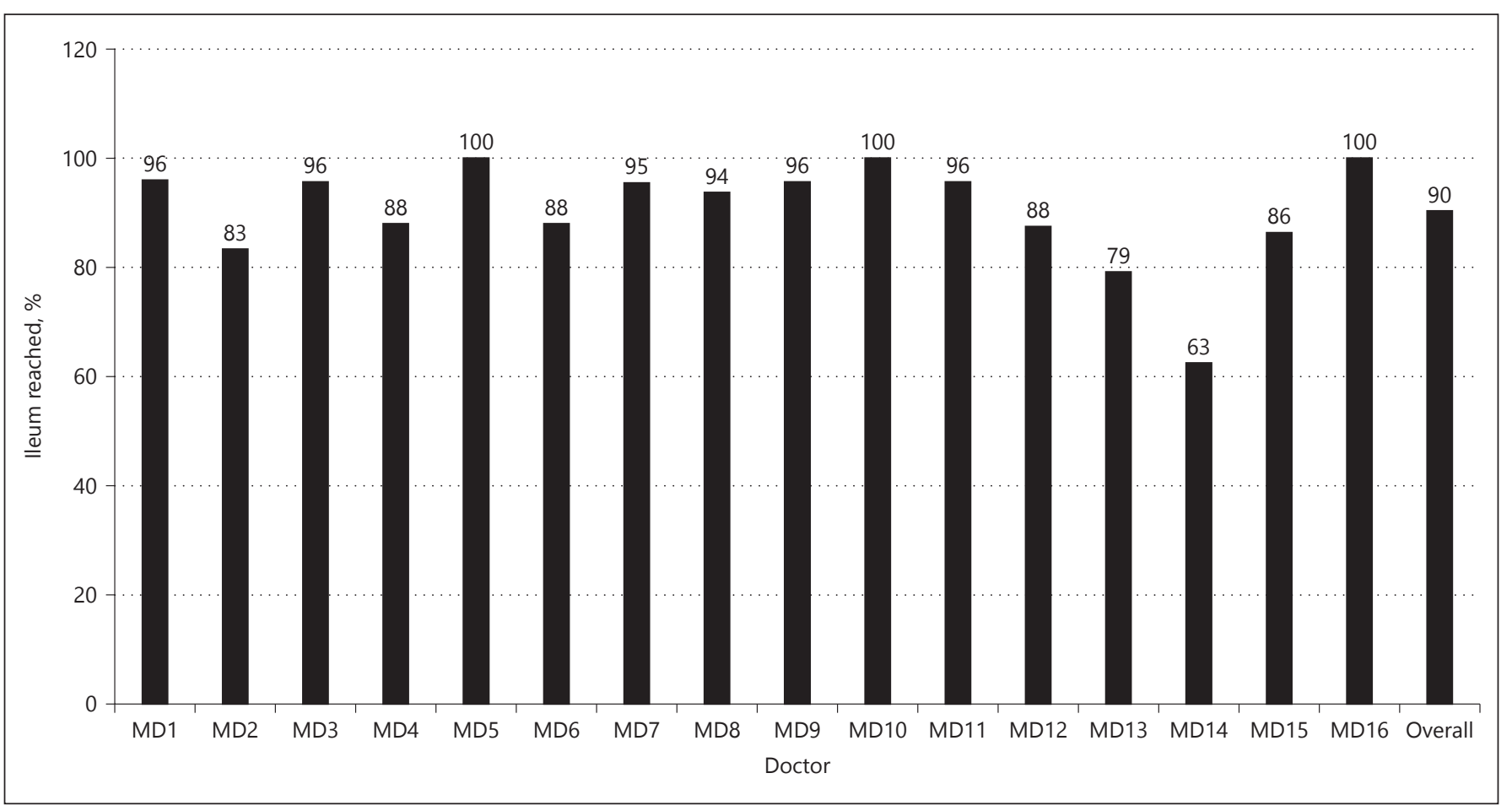

Fig. 4. Ileum cannulation rate by doctor. The ileal intubation rate varied considerably among the 16 individual physicians.

ed with a high diagnostic yield of $83 \%$. This finding is in agreement with many others showing the value of this test [16, 22-24]. In a study by Holtman et al. [22], the addition of fecal calprotectin to their models was the most useful test in determining the likelihood of IBD prior to endoscopy. In addition, calprotectin can be elevated in other colonic disorders including polyposis [25]. With the increasing use of biomarkers like calprotectin, it is anticipated that higher rates of diagnostic yield are likely. By monitoring diagnostic yields, individual providers may be able to alter their practice to more carefully select patients who will benefit from ileocolonoscopy.

This study showed that our center had a low serious complication rate; only 1 patient had an identified complication. In this patient, bleeding after polypectomy resulted in hospitalization, blood transfusion, and a repeat colonoscopy to control bleeding. Our low complication rate needs to be interpreted with caution in this retrospective study as some patients could have presented to outside emergency rooms or hospitals. Our complication rate is much lower than a recent report [26]. Kramer and Narkewicz [26] studied the frequency of adverse events that occurred within $72 \mathrm{~h}$ in a prospective observational pediatric cohort of 9,577 patients from a single center who underwent either (or both) upper and lower endoscopy. The overall adverse event rate was $2.6 \%$ with $1.7 \%$ of all cases requiring unanticipated medical care. For diagnostic colonoscopy, the study identified a $2.3 \%$ rate of adverse events which resulted in referral to the emergency department or an unanticipated evaluation by a physician; this severity of adverse event occurred in $9.4 \%$ of patients following polypectomy and included 4 of 128 who had a perforation.

Our study focused primarily on colonoscopy rather than EGD on diagnostic yield among clinicians primarily because EGD diagnostic yield is more difficult to interpret due to the high frequency of minor pathologic findings [20,21]. In our study, $<30 \%$ of esophagogastroduodenoscopies were considered normal both endoscopically and based on histology; this is in agreement with previous pediatric studies. In a systematic review of 1,871 children from 18 articles, Thakkar et al. [20] found that nonspecific histologic gastrointestinal inflammatory lesions varied between 23 and 93\%. Similarly, microscopic non-eosinophilic esophagitis has been noted in $20 \%$ of children and is more likely to indicate functional heartburn than nonerosive reflux disease [21].

The findings of this study have several limitations. First of all, this study included only patients who were 
healthy enough to have a colonoscopy in an ambulatory center. It is likely that the diagnostic yield would have been higher if hospitalized patients were included in this study. Second, with retrospective reviews, the quality of the data relies on adequate documentation. Some of the patients included may have had symptoms or laboratory studies that were not recorded. The definitions of charted clinical variables and endoscopy findings are not standardized. Even for terms like "diarrhea" each clinician may have a different definition. Additional limitations are related to the subjective nature of visual interpretation of endoscopic findings as well as the frequency and variability in the use of endoscopic biopsies. In addition, as is common in clinical practice, the histology was reviewed by only one pathologist for each patient; this could have led to both overinterpretation or underinterpretation of the findings. Finally, other parameters including bowel preparation and complications are more readily detected in prospective studies with standardized metrics. While a retrospective study has its limitations, one advantage is that the study would not have influenced individual clinicians in making decisions.

\section{Conclusions}

This study highlights the provider variability in diagnostic yield in an ambulatory pediatric gastroenterology private practice along with the variability in quality metrics like ileal intubation. In addition, this study confirms the value of calprotectin as predictor for higher diagnostic yield. This single-center retrospective study found diagnostic yields in excess of $40 \%$ and ileal intubation rates of $>90 \%$.

\section{Acknowledgment}

None.

\section{Statement of Ethics}

IRB approval was obtained for our retrospective chart review.

\section{Disclosure Statement}

The authors have no conflicts of interest to declare.

\section{Funding Sources}

Unfunded study.

\section{Author Contributions}

J.A.H.: participated with study design/concept, analysis/interpretation, drafting/revision of manuscript. J.F.: analyzed the data, ran the statistical programs, and developed the figures and tables. She helped write the statistical information in the manuscript. E.D.: participated with data acquisition and with analysis of data. J.D.L.: participated with study design/concept, helped analyze data, and provided critical review of draft/revision for important intellectual content.

\section{References}

1 Rex DK, Petrini JL, Baron TH, Chak A, Cohen J, Deal SE, et al. Quality indicators for colonoscopy. Gastrointest Endosc. 2006 Apr;63(4 Suppl):S16-28

2 Armstrong D. Quality indicators for colonoscopy: the road forward. Can J Gastroenterol Hepatol. 2014 May;28(5):242.

3 Kramer RE, Walsh CM, Lerner DG, Fishman DS. Quality Improvement in Pediatric Endoscopy: A Clinical Report From the NASPGHAN Endoscopy Committee. J Pediatr Gastroenterol Nutr. 2017 Jul;65(1):125-31.

4 Chassin MR, Loeb JM, Schmaltz SP, Wachter RM. Accountability measures-using measurement to promote quality improvement. $\mathrm{N}$ Engl J Med. 2010 Aug;363(7):683-8.

5 Bonilla S, Deli Wang, Saps M. The prognostic value of obtaining a negative endoscopy in chil- dren with functional gastrointestinal disorders. Clin Pediatr (Phila). 2011 May;50(5):396-401.

6 de Bie CI, Paerregaard A, Kolacek S, Ruemmele FM, Koletzko S, Fell JM, et al.; EUROKIDS Porto IBD Working Group of ESPGHAN. Disease phenotype at diagnosis in pediatric Crohn's disease: 5-year analyses of the EUROKIDS Registry. Inflamm Bowel Dis. 2013 Feb;19(2):378-85.

7 Kawada PS, O'Loughlin EV, Stormon MO, Dutt S, Lee CH, Gaskin KJ. Are We Overdoing Pediatric Lower Gastrointestinal Endoscopy? J Pediatr Gastroenterol Nutr. 2017 Jun; 64(6):898-902.

8 Manfredi MA, Jiang H, Borges LF, Deutsch AJ, Goldsmith JD, Lightdale JR. Good agreement between endoscopic findings and biopsy reports supports limited tissue sampling during pediatric colonoscopy. J Pediatr Gastroenterol Nutr. 2014 Jun;58(6):773-8.

9 Thomson M, Sharma S. Diagnostic Yield of Upper and Lower Gastrointestinal Endoscopies in Children in a Tertiary Centre. J Pediatr Gastroenterol Nutr. 2017 Jun;64(6):903-6.

10 Wang S, Younus O, Rawat D, Naik S, Giles E, Meadows N, et al. Clinical Presentation and Outcomes of Diagnostic Endoscopy in Newly Presenting Children With Gastrointestinal Symptoms. J Pediatr Gastroenterol Nutr. 2018 Jun;66(6):876-81.

11 Miele E, Giannetti E, Martinelli M, Tramontano A, Greco L, Staiano A. Impact of the Rome II paediatric criteria on the appropriateness of the upper and lower gastrointestinal endoscopy in children. Aliment Pharmacol Ther. 2010 Aug;32(4):582-90. 
$12 \mathrm{Wu}$ CT, Chen CA, Yang YJ. Characteristics and Diagnostic Yield of Pediatric Colonoscopy in Taiwan. Pediatr Neonatol. 2015 Oct; 56(5):334-8.

13 Thomson M, Eltumi M, Singh C, Eyles M, Shah N, Vadamalayan B, et al. Sub-10-minute High-quality Diagnostic Colonoscopy With Terminal Ileum Intubation in Children Is Feasible and Safe. J Pediatr Gastroenterol Nutr. 2019 Jul;69(1):6-12.

14 Noble AJ, Drouin E, Tamblyn R. Design of predictive models for positive outcomes of upper and lower gastrointestinal endoscopies in children and adolescents. J Pediatr Gastroenterol Nutr. 2008 Apr;46(4):409-13.

15 Lee WS, Zainuddin H, Boey CC, Chai PF. Appropriateness, endoscopic findings and contributive yield of pediatric gastrointestinal endoscopy. World J Gastroenterol. 2013 Dec; 19(47):9077-83.

16 Singh HK, Ee LC. Recurrent Abdominal Pain in Children: Is Colonoscopy Indicated? J Pediatr Gastroenterol Nutr. 2019 Feb;68(2):214-7.

17 Pasquarella CS, Kaplan B, Mahajan L, Lamparyk K, Kay M. A Single-center Review of Pe- diatric Colonoscopy Quality Indicators. J Pediatr Gastroenterol Nutr. 2019 May;68(5): 648-54.

18 Thakkar K, Holub JL, Gilger MA, Shub MD, McOmber M, Tsou M, et al. Quality indicators for pediatric colonoscopy: results from a multicenter consortium. Gastrointest Endosc. 2016 Mar;83(3):533-41.

19 Sheiko MA, Feinstein JA, Capocelli KE, Kramer RE. Diagnostic yield of EGD in children: a retrospective single-center study of 1000 cases. Gastrointest Endosc. 2013 Jul; 78(1):47-54.e1.

20 Thakkar K, Gilger MA, Shulman RJ, El Serag HB. EGD in children with abdominal pain: a systematic review. Am J Gastroenterol. 2007 Mar;102(3):654-61.

21 Mahoney LB, Nurko S, Rosen R. The Prevalence of Rome IV Nonerosive Esophageal Phenotypes in Children. J Pediatr. 2017 Oct; 189:86-91.

22 Holtman GA, Lisman-van Leeuwen Y, Day AS, Fagerberg UL, Henderson P, Leach ST, et al. Use of Laboratory Markers in Addition to Symptoms for Diagnosis of Inflammatory
Bowel Disease in Children: A Meta-analysis of Individual Patient Data. JAMA Pediatr. 2017 Oct;171(10):984-91.

23 Yang Z, Clark N, Park KT. Effectiveness and cost-effectiveness of measuring fecal calprotectin in diagnosis of inflammatory bowel disease in adults and children. Clin Gastroenterol Hepatol. 2014 Feb;12(2):25362.e2.

24 Heida A, Holtman GA, Lisman-van Leeuwen Y, Berger MY, van Rheenen PF. Avoid Endoscopy in Children With Suspected Inflammatory Bowel Disease Who Have Normal Calprotectin Levels. J Pediatr Gastroenterol Nutr. 2016 Jan;62(1):47-9.

25 Olafsdottir I, Nemeth A, Lörinc E, Toth E, Agardh D. Value of Fecal Calprotectin as a Biomarker for Juvenile Polyps in Children Investigated With Colonoscopy. J Pediatr Gastroenterol Nutr. 2016 Jan;62(1):43-6.

26 Kramer RE, Narkewicz MR. Adverse Events Following Gastrointestinal Endoscopy in Children: Classifications, Characterizations, and Implications. J Pediatr Gastroenterol Nutr. 2016 Jun;62(6):828-33. 